\title{
General Psychiatry Development and challenges of mental health in China
}

To cite: Que J, Lu L, Shi L. Development and challenges of mental health in China. General Psychiatry 2019;32:e100053. doi:10.1136/ gpsych-2019-100053

Received 18 January 2019 Accepted 20 January 2019

Check for updates

(C) Author(s) (or their employer(s)) 2019. Re-use permitted under CC BY-NC. No commercial re-use. See rights and permissions. Published by BMJ.

Peking University Sixth Hospital, Peking University Institute of Mental Health, NHC Key Laboratory of Mental Health (Peking University), National Clinical Research Center for Mental Disorders (Peking University Sixth Hospital), Peking University, Beijing, China

Correspondence to

Professor Lin Lu;

linlu@bjmu.edu.cn
With the socioeconomic development and the acceleration of the ageing process of the population, the incidence rates of mental disorders and psycho-behavioural problems have become higher and higher worldwide. So far, the disease burden caused by mental disorders has ranked second in the world. ${ }^{1}$ In China, the disease burden caused by mental disorders accounts for $13 \%$ of all non-communicable diseases burden. ${ }^{2}$ Hence, mental health has become a major public health problem and social problem. In order to promote mental health, the Chinese government has promulgated a series of policies and regulations, as well as reform measures. Moreover, the upcoming 'China Brain Project' will also focus on some mental disorders such as autism, depression and dementia to improve mental health research in China.

\section{CURRENT STATUS OF MENTAL HEALTH IN CHINA \\ Mental health services \\ High prevalence of mental disorders and low \\ consultation rate}

The epidemiological survey conducted in four provinces in China showed that the prevalence of mental disorders among adults in China was $17.5 \%$, in which the prevalence of mood disorders (mainly depression), anxiety disorders and substance use disorders were $6.1 \%, 5.6 \%$ and $5.9 \%$, respectively. ${ }^{3}$ According to the WHO report, the recognition rate of global mental disorders is around $50 \%$, and the recognition rate in China is far below the world average. Taking depression as an example, the recognition rate of depression was only $21 \%$ in Shanghai, China. In addition, the rate of diagnosis and treatment of mental disorders is also relatively low, with an average of only around 150 people per 100000 people receiving treatment for serious mental disorders. The treatment rate for serious mental disorders in high-income countries is about 17 times that of low-income countries. ${ }^{4}$

\section{High proportion of refractory mental disorders and} poor medications response

The effectiveness of medications treating mental disorders varies among patients.
For example, when one patient with schizophrenia treated with aripiprazole gains improvement, about four patients cannot benefit from the same medication. When duloxetine is effective in treating one patient with depression, it is instead ineffective for the other eight patients. ${ }^{5}$ For the sake of improving the precision of mental illness diagnosis and the effectiveness of various treatments, the US National Institute of Mental Health proposed the Research Domain Criteria programme in $2008 .{ }^{6}$

\section{Insufficient and unevenly distributed mental health resources}

In China, there is insufficient mental health resources and service capacity: (1) financial investment-the per capita investment from the Chinese government for psychiatric hospitals is about US $\$ 1.07,{ }^{7}$ which is far lower than US\$35.06 in high-income countries during the same period $^{8}$; (2) hospital beds-the number of psychiatric beds in China per 10000 population is $3.15,{ }^{9}$ which is far lower than the 7.13 beds in high-income countries ${ }^{8}$; (3) professionalsin China for every 100000 population, there are 2.19 psychiatric physicians (including assistant practitioners) and 5.51 registered nurses, ${ }^{9}$ whereas in high-income countries, there are 13.06 psychiatrists and 23.49 registered nurses per every 100000 people. $^{8}$ At the same time, we are facing problems such as having difficulties in bringing in mental health field talents and lacking vocational rehabilitation technicians. Moreover, the distribution of China's mental health resources is unbalanced, with the most hospitals and professionals concentrated in provincial capitals and developed eastern regions. It is indicated that $47.21 \%$ of institutions, $42.06 \%$ of psychiatric beds, $48.65 \%$ of physicians and $45.25 \%$ of nurses are located in 11 eastern provinces. Moreover, mental health personnel in grassroots medical institutions has insufficient service capacity, and most of the personnel are part-time. 


\section{Progress in mental disorder research}

\section{Autism spectrum disorder}

Autism spectrum disorder is a kind of neurodevelopmental disorders characterised by trouble with social interaction, impaired communication, restricted interest and repetitive behaviour. The aetiology and pathogenesis are still unclear while generally thought that autism spectrum disorder is closely related to genetic factors. Liu et al. from the Chinese Academy of Sciences found that MECP2 transgenic monkeys showed repetitive behaviours and social interaction impairments ${ }^{10}$ and established the world's first non-human primate model of autism which is significant for exploring pathogenesis and possible interventions for autism. The research team from Central South University discovered the autism susceptibility genes through genome-wide association study, ${ }^{11}$ molecular inversion probes, ${ }^{12}$ and targeted sequencing and functional analysis ${ }^{13}$ which provides the possibility of early diagnosis and treatment of autism.

\section{Major depressive disorder}

Depression is one of the most common mental disorders, and it has placed a heavy burden on the society and families. However, its pathogenesis is yet completely clear. Cai et al. found two loci that contribute to the risk of major depressive disorder on chromosome 10: one near the SIRT1 gene, the other in an intron of the LHPP gene using low-coverage whole-genome sequencing of 5303 Chinese women with recurrent major depressive disorder. ${ }^{14}$ Besides, Researchers from Zhejiang University proposed a new rapid antidepressant mechanism of ketamine which showed that blockade of NMDAR-dependent bursting activity in the lateral habenula relieved the inhibition of the reward. ${ }^{15}$ Based on this finding, they also pointed out that astroglial potassium channel 4.1 (Kir4.1) could also exert rapid antidepressant effects. ${ }^{16}$ In addition, our team found that depression promoted the coupling of death-associated protein kinase 1 (DAPK1) with the glutamate receptor subunit NR2B in the prefrontal cortex, and we proposed a new hypothesis that the interaction of extracellular DAPK1 with the NMDAR GluN2B mediated rapid effects of antidepressant. ${ }^{17}$ Meanwhile, we also found that depression would lead to down-regulation of the protein kinase PKM $\zeta$ signalling pathway in the prefrontal cortex, and activation of this signalling pathway could produce antidepressant effects. ${ }^{18}$ These studies have promoted the understanding of the pathogenesis of depression and provided a number of new molecular targets for the development of new antidepressants.

\section{Alzheimer's disease}

With the acceleration of population ageing process, the number of patients with Alzheimer's Disease (AD) has risen sharply which makes it as the brain disease with the fastest-growing disease burden. Researchers from the Army Medical University found that peritoneal dialysis could effectively remove amyloid beta $(\mathrm{A} \beta)$ from the blood and might also decrease brain $A \beta$ deposition to improve cognition. ${ }^{19}$ Meanwhile, they also innovatively used transgenic AD mice in parallel with their wild-type littermates to connect the two blood circulations. The study showed that $A \beta$ derived from transgenic mice could induce $\mathrm{AD}$ pathological changes through the blood into the brain of normal mice and could cause functional deficits of neurons, ${ }^{20}$ indicating the important role of $\mathrm{A} \beta$ in the development of $\mathrm{AD}$. In addition, we did a meta-analysis which found that there was a high risk of incident all-cause dementia in individuals with sleep disturbances, ${ }^{21}$ and this might be probably caused by inadequate brain cleansing of neurotoxic waste products during sleep. $^{22}$ The finding provides a theoretical basis for understanding the pathogenesis of $\mathrm{AD}$ and contributes to the early prevention of the disease.

\section{Pathological emotional memory related diseases}

Learning and memory are essential for the survival of an individual, but pathological emotional memory can lead to a series of mental disorders such as drug addiction, post-traumatic stress disorder, anxiety disorders and so forth. However, there is still no effective interventions for treating pathological emotional memory related disorders. Our research team innovatively proposed that the 'conditioned stimulus memory retrieval-extinction' procedure could erase drug memory, that is, extinction during the time window of reconsolidation after retrieval of a contextual cue could erase the cue-associated drug memory, and attenuate drug-seeking behaviour to eliminate pathological addictive memory. ${ }^{23}$ On this basis, we also proposed the 'unconditioned stimulus memory retrieval-extinction' strategy to effectively interrupt the pathological memories related to all cues. ${ }^{24-26}$ Importantly, we successfully achieved clinical transformation of this theory, for example, applying propranolol to interfere unconditioned stimulus-induced memory reconsolidation process could decrease nicotine craving in smokers. ${ }^{27}$ Furthermore, in the awake state, when treating pathological emotional memory-related diseases, patients needed to repeatedly re-experience traumatic events which was not conducive to treatment compliance. To overcome this drawback, we found that conditioned stimulus re-exposure during slow wave sleep promoted fear memory extinction without altering sleep profiles which may be a new way to eliminate negative emotional memory without pain. ${ }^{28}$ This series of original work overcomes the limitations of existing treatments and is a breakthrough in the field of pathological emotional memory related diseases treatment.

\section{Sleep disorders}

Sleep is critical for growth and development; however, the mechanism underlying sleep is still unknown. Ren et al. from the Army Medical University found that the paraventricular thalamus was the key nucleus to regulate the sleep and wakefulness process, and suppression of the paraventricular thalamus neuronal activity caused a reduction in wakefulness, whereas activation of paraventricular thalamus neurons induced a rapid transition from sleep to wakefulness. ${ }^{29}$ Moreover, recent studies indicated that sleep was close to mental health. Our meta-analysis research found that the prevalences of sleep disorders and depressive disorders were higher in the community of the elderly population 
( $\geq 60$ years), and the comorbidities of sleep disturbances and depression were common. ${ }^{30}$ With regard to its possible mechanism, the research team from Fudan University ${ }^{31}$ discovered that lateral orbitofrontal cortex, dorsal prefrontal cortex, cingulate cortices and insula were potential neural basis for the association between depression and poor sleep quality. In future, more studies are needed to reveal the reason between sleep disorders and other disease.

\section{Prospects of mental health in China}

Currently, under the concept of the biological-psychology-sociological medicine pattern, mental health has attracted more attention, and its service in China has been greatly expanded to include not only the severe mental disorders, such as schizophrenia and bipolar disorder, but also depression, anxiety, maladaptation, and other mental and psychological problems. Moreover, the transformation of the disease philosophy, the innovation of basic medical theory and the rapid advancement of new technology will bring milestones development to psychiatry medicine. In 2013, the implementation of the Mental Health Law in China made the clinical research and medical services for mental disorders law-abiding and rule-based. In 2014, China established the National Clinical Research Center for Mental Disorders which raised the mental and psychological development to the national strategic level for the first time. In addition, 'Healthy China 2030 Planning Outline', '13th Five-Year Hygiene and Health Plan', 'China's Long-Term Plan for Prevention and Treatment of Chronic Diseases 2017-2025' and 'National Mental Health Work Plan 20152020 ' are announced gradually. These announcements have indicated the urgent need for strengthening mental health research, service and management in China and put forth new requirements for the future development of psychiatry.

\section{Depth exploration of the pathogenesis in mental disorders}

With rapid development of neuroscience technology and the innovation of methodology in recent years, the combined application of imaging technologies, molecular biology, and information science and engineering will deeply explain the pathogenesis of mental disorders at different levels. The rapid development of genetic techniques such as highthroughput sequencing enables us to detect new susceptibility genes associated with mental disorders; neuronal circuit manipulation techniques such as optogenetics, chemical inheritance and tracing of neural loops, as well as rapid imaging techniques and analytical methods development are helpful to make the mechanisms of mental disorders clear from neuronal circuits to brain regions. Animal models of mental disorders such as non-human primates and induced pluripotent stem cells provide the possibility for explaining the pathogenesis of mental disorders deeply.

\section{Achieving early diagnosis and treatment of mental disorders}

The cause of mental disorders is complex, and the course of disease is protracted. In the future, efforts should be made to develop new technologies that can be used for early prevention, early identification and early intervention, thereby effectively reducing the incidence, progression and relapse of mental disorders. First, we should promote the integration of multidisciplinary, such as neurology, sleep medicine, immunology and psychiatry. And on the basis of biological big data and artificial intelligence, a large cohort should be established to construct an integrated intelligent system for mental disorder screening, diagnosis, treatment and rehabilitation. Second, the interaction of genetic and environmental risk factors in the development of mental disorders should be explored to identify high-risk groups for precise prevention and reduce the occurrence of the diseases by controlling risk factors. Third, current classification diagnosis of mental disorders mainly depends on the symptoms and signs of patients and lacks objective indicators that can be used for early screening of high-risk groups with high reliability and validity. In the future, biological indicators will be intended for redefining and classifying mental disorders to provide personalised treatment options for patients. Fourth, with the deepening of research, more targets related to mental disorders have been discovered. In future, the new-generation psychiatric medications with quick onset, good efficacy and few side effects should be developed. In addition, it is necessary to apply non-medication interventions such as physical therapy and psychotherapy to treat mental disorders.

\section{Building a sound mental health service system}

At present, the construction of mental health service system in China is relatively lagging. Community-based rehabilitation system is an important part of the mental health service system. Making up the insufficiency in mental health rehabilitation system is important to prevent disability and poverty caused by mental disorders and promote the re-entry of patients to the society. Also, private mental hospitals have arbitrary charges, false medical advertisements and unqualified medical personnel problems. It is beneficial to standardise the development of private hospitals. In addition, the mental health service system still has problems such as inadequate healthcare network, ignoring the importance of disease prevention, and lacking sound regulations and policy support for mental health work. Therefore, relevant policies and regulations should be made to promote the changes in mental health service, establish a mental health alliance and provide the best quality services for people with mental disorders.

Overall, the medical health system reform in China has entered a critical period, and there are challenges and opportunities for us to develop mental health. Regarding the increasing burden of mental disorders, shortage of mental health resources and the numerous predicaments faced by present condition of mental disorder prevention and treatment, our country should increase investment in the field of mental health, raise public awareness of mental health through education, build mental health rehabilitation system in the whole country and improve the accessibility of mental health services. Clinical workers and researchers should cooperate with each other to strengthen the understanding of the pathogenesis of mental disorders 
and improve the level of medical services. Only in this way can bring the mental health achieve faster and better development, and the physical and mental health of people and the harmony and stability of society can be effectively guaranteed.

Contributors LL proposed the topic and main idea, JQ wrote the draft of the manuscript, LL and LS commented on and revised the manuscript. All authors contributed to and have approved the final manuscript.

Funding The authors have not declared a specific grant for this research from any funding agency in the public, commercial or not-for-profit sectors.

Competing interests None declared.

Patient consent for publication Not required.

Provenance and peer review Not commissioned; internally peer reviewed.

Open access This is an open access article distributed in accordance with the Creative Commons Attribution Non Commercial (CC BY-NC 4.0) license, which permits others to distribute, remix, adapt, build upon this work non-commercially, and license their derivative works on different terms, provided the original work is properly cited and the use is non-commercial. See: http://creativecommons.org/ licenses/by-nc/4.0

\section{REFERENCES}

1. James SL, Abate D, Abate $\mathrm{KH}$, et al. Global, regional, and national incidence, prevalence, and years lived with disability for 354 diseases and injuries for 195 countries and territories, 1990-2017: a systematic analysis for the global burden of Disease Study 2017. The Lancet 2018;392:1789-858.

2. Charlson FJ, Baxter AJ, Cheng HG, et al. The burden of mental, neurological, and substance use disorders in China and India: a systematic analysis of community representative epidemiological studies. The Lancet 2016;388:376-89.

3. Phillips MR, Zhang J, Shi Q, et al. Prevalence, treatment, and associated disability of mental disorders in four provinces in China during 2001-05: an epidemiological survey. The Lancet 2009;373:2041-53.

4. WHO. World mental health atlas, 2014.

5. Schork NJ. Personalized medicine: time for one-person trials. Nature 2015;520:609-11.

6. Health NloM, 2008. Research domain criteria (RDoC). Available: https://www.nimh.nih.gov/research-priorities/rdoc/index.shtml

7. National Health and Family Planning Commission. China health and family planning statistical Yearbook 2017. Beijing: China Union Medical University Press, 2017.

8. WHO. World mental health atlas, 2017.

9. Shi $\mathrm{CH}$, Ma N. Analysis of Mental Health Resources in China in 2015. In: Papers of the 16th national psychiatric conference of the Chinese medical association, 2018.

10. Liu Z, Li X, Zhang JT, et al. Autism-like behaviours and germline transmission in transgenic monkeys overexpressing MeCP2. Nature 2016;530:98-102.
11. Xia K, Guo H, Hu Z, et al. Common genetic variants on 1 p13.2 associate with risk of autism. Mol Psychiatry 2014;19:1212-9.

12. Wang $\mathrm{T}$, Guo H, Xiong B, et al. De novo genic mutations among a Chinese autism spectrum disorder cohort. Nat Commun 2016;7.

13. Li J, Wang L, Guo H, et al. Targeted sequencing and functional analysis reveal brain-size-related genes and their networks in autism spectrum disorders. Mol Psychiatry 2017;22:1282-90.

14. Cai N, Bigdeli TB, Kretzschmar W, et al. Sparse whole-genome sequencing identifies two loci for major depressive disorder. Nature 2015;523:588-91.

15. Yang $Y$, Cui $Y$, Sang $K$, et al. Ketamine blocks bursting in the lateral habenula to rapidly relieve depression. Nature 2018;554:317-22.

16. Cui Y, Yang Y, Ni Z, et al. Astroglial Kir4.1 in the lateral habenula drives neuronal bursts in depression. Nature 2018;554:323-7.

17. Li SX, Han Y, Xu LZ, et al. Uncoupling Dapk1 from NMDA receptor GluN2B subunit exerts rapid antidepressant-like effects. Mol Psychiatry 2018;23:597-608.

18. Yan W, Liu JF, Han Y, et al. Protein kinase M $\mathrm{\zeta}$ in medial prefrontal cortex mediates depressive-like behavior and antidepressant response. Mol Psychiatry 2018;23:1-14.

19. Jin WS, Shen LL, Bu XL, et al. Peritoneal dialysis reduces amyloidbeta plasma levels in humans and attenuates Alzheimer-associated phenotypes in an APP/PS1 mouse model. Acta Neuropathol 2017;134:207-20.

20. XL B, Xiang Y, Jin WS, et al. Blood-derived amyloid-beta protein induces Alzheimer's disease pathologies. Molecular psychiatry 2017;23:1-9.

21. Shi L, Chen SJ, Ma MY, et al. Sleep disturbances increase the risk of dementia: a systematic review and meta-analysis. Sleep Med Rev 2018;40:4-16.

22. Xie L, Kang H, Xu Q, et al. Sleep drives metabolite clearance from the adult brain. Science 2013;342:373-7.

23. Xue $\mathrm{YX}$, Luo $\mathrm{YX}$, Wu $\mathrm{P}$, et al. A memory retrieval-extinction procedure to prevent drug craving and relapse. Science 2012;336:241-5.

24. Liu J, Zhao L, Xue Y, et al. An unconditioned stimulus retrieval extinction procedure to prevent the return of fear memory. Biol Psychiatry 2014;76:895-901.

25. Luo Y-xiao, Xue Y-xue, Liu J-feng, et al. A novel UCS memory retrieval-extinction procedure to inhibit relapse to drug seeking. Nature Communications 2015;6.

26. Xue YX, Chen YY, Zhang LB, et al. Selective inhibition of amygdala neuronal ensembles encoding nicotine-associated memories inhibits nicotine preference and relapse. Biol Psychiatry 2017;82:781-93.

27. Xue YX, Deng JH, Chen YY, et al. Effect of selective inhibition of reactivated nicotine-associated memories with propranolol on nicotine craving. JAMA Psychiatry 2017;74:224-32.

28. He J, Sun HQ, Li SX, et al. Effect of conditioned stimulus exposure during slow wave sleep on fear memory extinction in humans. Sleep 2015;38:423-31.

29. Ren S, Wang Y, Yue F, et al. The paraventricular thalamus is a critical thalamic area for wakefulness. Science 2018;362:429-34.

30. Bao YP, Han Y, Ma J, et al. Cooccurrence and bidirectional prediction of sleep disturbances and depression in older adults: meta-analysis and systematic review. Neurosci Biobehav Rev 2017;75:257-73.

31. Cheng W, Rolls ET, Ruan H, et al. Functional connectivities in the brain that mediate the association between depressive problems and sleep quality. JAMA Psychiatry 2018;75:1052-61.

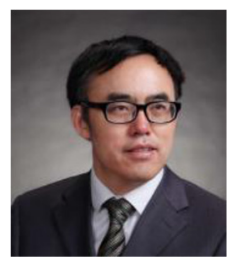

Lin Lu, Academician of Chinese Academy of Sciences, Director of the Sixth Hospital of Peking University, Dean of the Mental Health Institute of Peking University, Director of the National Center for Clinical Psychiatric Diseases Research. His research interests include the study of the pathogenesis and intervention strategies of psychiatric disorders and sleep disorders. 